\title{
Prediction of a CDMA Output Spectrum Based on Intermodulation Products of Two-Tone Test
}

\author{
Seung-June Yi, Sangwook Nam, Member, IEEE, Sung-Hoon Oh, and Jae-Hee Han, Student Member, IEEE
}

\begin{abstract}
A prediction method of a code-division multiple-access (CDMA) output spectrum based on intermodulation (IM) products of a two-tone test is proposed in this paper. An RF power amplifier is mathematically modeled by a complex envelope transfer function and, using this model, analytical expressions of IM products and a CDMA output spectrum are derived, respectively. By combining these two expressions, we finally derive an analytical expression that relates IM products to a CDMA output spectrum. It is shown in this expression that not only AM-AM, but also AM-PM distortion affects the CDMA output spectrum. Comparison between the measured and predicted results shows that the output spectrum predicted by this method agrees well with the measured spectrum.
\end{abstract}

Index Terms-AM-AM distortions, AM-PM distortions, CDMA output spectrum, complex envelope transfer function, IM products, RF power amplifier, two-tone test.

\section{INTRODUCTION}

$\mathbf{T}$ HE recent digital mobile communication systems use a code-division multiple-access (CDMA) scheme for high spectral efficiency. When a CDMA signal is passed through an $\mathrm{RF}$ transmitter, however, spectral regrowth is inevitably generated by nonlinear devices, and the spectral efficiency gained by using a CDMA scheme is reduced. Since the spectral regrowth is stringently regulated and is mostly generated by a nonlinear RF power amplifier, it is very important for RF system designers to predict the distortion effects of power amplifiers on CDMA signals. To this end, many techniques have been developed, each providing good results. Most of them build the baseband equivalent mathematical model of a nonlinear power amplifier based on the quadrature decomposition technique that was first proposed by Kaye [1]. The spreading signal is then put into the power amplifier's mathematical model and, by using a fast Fourier transform (FFT) algorithm, the amplified CDMA output spectrum is calculated [2]-[4]. Though this method predicts the output spectrum accurately, it is quite difficult to see how a power amplifier's AM-AM and AM-PM distortions affect the spectral regrowth. An analytical approach is developed by $\mathrm{Wu}$ [5]-[7], where the stochastic theory is combined to the quadrature decomposition technique. In that paper, an analytical

Manuscript received July 9, 1999; revised December 17, 1999.

S.-J. Yi is with the Advanced Telecommunication Research Department, Advanced Telecommunication Research Laboratory, LG Electronics Inc., 431-080 Anyang, Korea and is also with the School of Electrical Engineering, Seoul National University, 151-742 Seoul, Korea (e-mail: ysj@1gic.co.kr).

S. Nam, and J.-H. Han are with the School of Electrical Engineering, Seoul National University, 151-742 Seoul, Korea (e-mail: snam@ee.snu.ac.kr; jhhan@inmac3.snu.ac.kr).

S.-H. Oh is with Next Generation Communication Laboratory, LG Electronics Inc., 431-080 Anyang, Korea (e-mail: odori@1gic.co.kr).

Publisher Item Identifier S 0018-9480(01)03309-9.

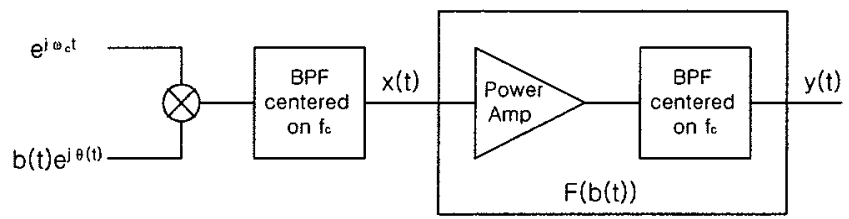

Fig. 1. Block diagram of a general transmit system.

expression of the amplified CDMA output spectrum is derived by AM-AM distortion, and the power amplifier's traditional nonlinearity parameter interception point (IP) is directly related to the output spectrum. Unfortunately, to the author's knowledge, the relationship between intermodulation (IM) products of a two-tone test and a CDMA output spectrum has not been reported. Though the IP is closely related to IM, the IP is a scalar parameter, and Wu's paper cannot explain the effects of AM-PM distortions on the CDMA output spectrum. Since IM is a vector process, as is shown in [8] and [9], not only AM-AM, but also AM-PM distortion should be considered in predicting the amplified CDMA output spectrum.

This paper presents an analytical expression that relates IM products to a CDMA output spectrum. With this expression, one can predict the CDMA output spectrum only by IM products of a two-tone test. Since the measurement of the amplified CDMA output spectrum requires the spreading input signal that is generated from the baseband and modem part, it is much easier to measure the IM products of a two-tone test than the amplified CDMA output spectrum. Moreover, the analytical expression that we derived can give the increased insight into explaining why adjacent channel power (ACP) and IM do not necessarily track each other. The reason is that the IM phase does affect the spectral regrowth. To show the effects of AM-PM distortion on the CDMA output spectrum, a nonlinear power amplifier is modeled by a complex envelope transfer function not only by AM-AM distortion. A verification is performed with a wide-band CDMA (WCDMA) system, where the measured and predicted spectrums are compared.

\section{Mathematical Model of AN RF POWer AmPlifier}

The general transmit system can be simplified as Fig. 1, where a nonlinear power amplifier is sandwiched between bandpass filters (BPFs). In Fig. 1, the input signal is of the form

$$
\begin{aligned}
x(t) & =b(t) \cos \left(\omega_{c} t+\theta(t)\right) \\
& =\operatorname{Re}\left[b(t) e^{j \theta(t)} e^{j \omega_{c} t}\right] \\
& \triangleq \operatorname{Re}\left[x_{o}(t) e^{j \theta(t)} e^{j \omega_{c} t}\right]
\end{aligned}
$$


where $b(t)$ is an amplitude and $\theta(t)$ is a phase of the carrier, respectively, $\omega_{c}$ is the carrier angular frequency, and $x_{o}(t)$ denotes the baseband equivalent input signal. Due to the nonlinearities of the power amplifier, the input signal experiences AM-AM and AM-PM distortions, and the output signal can be represented as

$$
\begin{aligned}
y(t) & =\operatorname{Re}\left[y_{o}(t) e^{j \theta(t)} e^{j \omega_{c} t}\right] \\
& \triangleq \operatorname{Re}\left[F(b(t)) e^{j \theta(t)} e^{j \omega_{c} t}\right] \\
& =|F(b(t))| \cos \left(\omega_{c} t+\theta(t)+\angle F(b(t))\right)
\end{aligned}
$$

where $y_{o}(t)$ denotes the baseband equivalent output signal and $F(b(t))$ represents the complex envelope transfer function of the power amplifier. In (2), $|F(b(t))|$ is equivalent to AM-AM distortion and $\angle F(b(t))$ to AM-PM distortion. For a narrow-band memoryless system, $F(b(t))$ function can be represented by the odd-order Taylor series as [4]-[7]

$F(b(t))=f_{1} b(t)+f_{3} b^{3}(t)+f_{5} b^{5}(t)+\cdots=\sum_{k=1}^{\infty} f_{2 k-1} b^{2 k-1}(t)$

since the even-order terms are filtered out by the second BPF. The complex coefficients $f_{2 k-1}$ can be obtained from the odd-order complex polynomial fitting of the complex envelope transfer function. Note that each phase of $f_{2 k-1}$ has a different value, which means that each order of distortion experiences different $\mathrm{AM}-\mathrm{AM}$ and $\mathrm{AM}-\mathrm{PM}$ distortions. In many papers, the complex nonlinearity $F(b(t))$ is quadratically decomposed into AM-AM and AM-PM distortions as $F(b(t))=M(b(t)) e^{j P(b(t))}$, but, in this case, since $e^{j P(b(t))}$ also has the amplitude quantity in addition to the phase quantity, it is difficult to represent each order of distortion. That is, if, for the most simple example, $M(b(t))=m_{1} b(t)$, $P(b(t))=p_{1} b(t)$, and $b(t)=\cos \omega t$, then

$$
\begin{aligned}
M(b(t)) e^{j P(b(t))} & =m_{1} \cos \omega t e^{j p_{1} \cos \omega t} \\
& =\tilde{m}_{1} e^{j \tilde{p}_{1}} \cos \omega t+\tilde{m}_{3} e^{j \tilde{p}_{3}} \cos 3 \omega t+\cdots
\end{aligned}
$$

and it generates every odd-order harmonic components. This effect is graphically shown in Fig. 2, where the waveform of $\cos \omega t e^{j p_{1} \cos \omega t}$ is plotted with various $p_{1}$. It is clearly shown in Fig. 2 that, for small values of $p_{1}$, the waveform is almost a pure sine wave, but when $p_{1}$ grows large, the amplitude is greatly distorted by phase distortions and the waveform no longer shapes the sine wave. If we use FFT algorithm, this effect can be fully described and the output spectrum can be correctly predicted, but an analytical expression of the output spectrum is hard to derive since it is very difficult to derive the general expressions of $\tilde{m}_{2 k-1}$ and $\tilde{p}_{2 k-1}$. Therefore, we use the complex envelope transfer function $F(b(t))$, by which an analytical expression of output spectrum can be easily obtained. For $F(b(t)), m_{1} \cos \omega t e^{j p_{1} \cos \omega t}$ can be represented as

$$
\begin{aligned}
m_{1} \cos \omega t e^{j p_{1} \cos \omega t} & =f_{1} \cos \omega t+f_{3} \cos { }^{3} \omega t+\cdots \\
& =y_{1} \cos \omega t+y_{3} \cos 3 \omega t+\cdots
\end{aligned}
$$

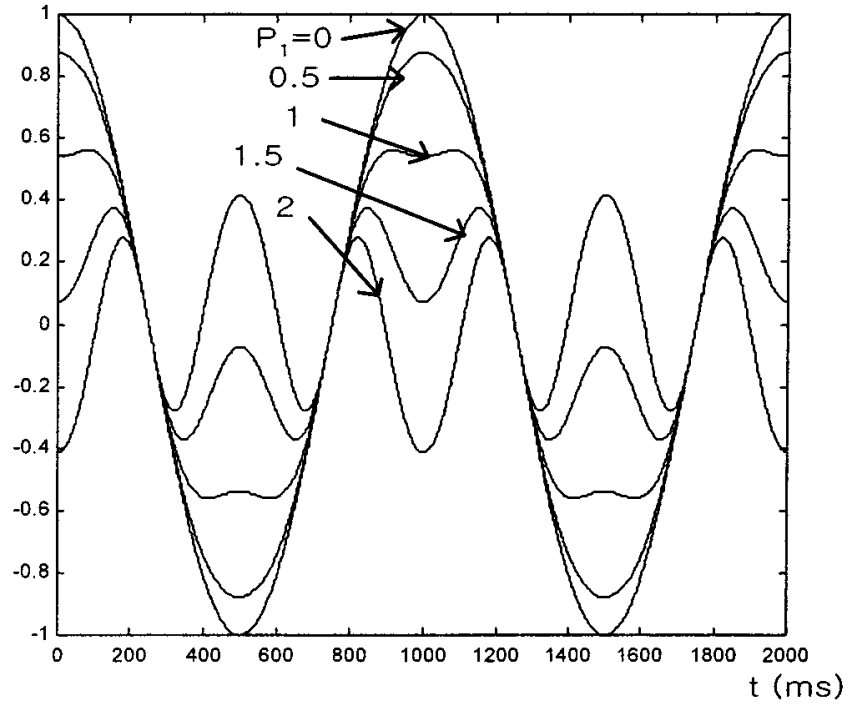

Fig. 2. Waveform of $\cos \omega t e^{j p_{1}} \cos \omega t$ with various $p_{1}$ values.

and the general expression of $y_{2 k-1}$ is easily obtained, as will be shown in the following section. Without loss of generality, $F(b(t))$ can be characterized by first $n$ terms, and we will use the following as a baseband equivalent model of an RF power amplifier:

$$
y_{o}(t)=F\left(x_{o}(t)\right)=\sum_{k=1}^{n} f_{2 k-1} x_{o}^{2 k-1}(t)
$$

\section{ANALYTICAL EXPRESSION OF IM PRODUCTS IN CONTINUOUS-WAVE Two-TONE INPUT}

In a two-tone test, IM products are inevitably generated from the power amplifier's nonlinearity. Generally, $\mathrm{IM}_{3}$ is used as a linearity parameter, but when an input signal becomes large, higher order IM products are also generated. Therefore, we derive an analytical expression of IM products in general order. The input signal in a two-tone test can be represented as

$$
\begin{aligned}
x(t) & =\frac{s}{2} \cos \left(\left(\omega_{c}+\Delta \omega\right) t+\theta(t)\right)+\frac{s}{2} \cos \left(\left(\omega_{c}-\Delta \omega\right) t+\theta(t)\right) \\
& =s \cos (\Delta \omega t) \cos \left(\omega_{c} t+\theta(t)\right) \\
& =\operatorname{Re}\left[s \cos (\Delta \omega t) e^{j \theta(t)} e^{j \omega_{c} t}\right]
\end{aligned}
$$

where $s / 2$ is an amplitude of each tone. The total input power of this signal is

$$
P_{i}=2 \times \frac{1}{2}\left(\frac{s}{2}\right)^{2} \frac{1}{R}=\frac{s^{2}}{4 R}(W)
$$

where $R$ is the nominal impedance usually $50 \Omega$. By comparing

$$
x_{o}(t)=s \cos (\Delta \omega t)
$$




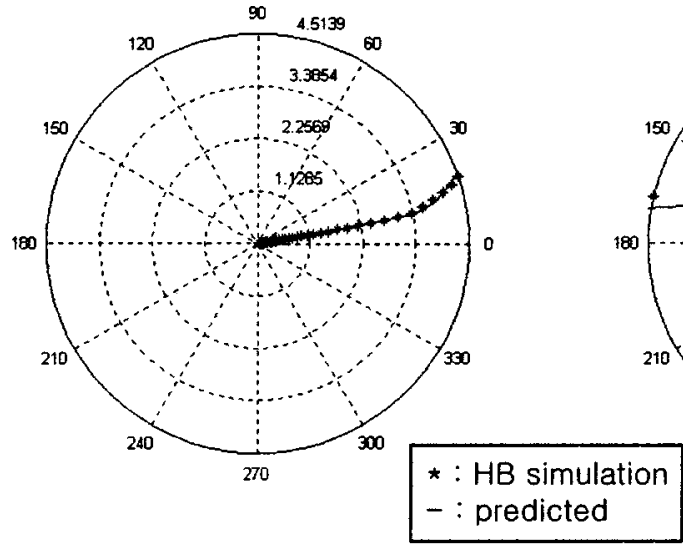

(a)

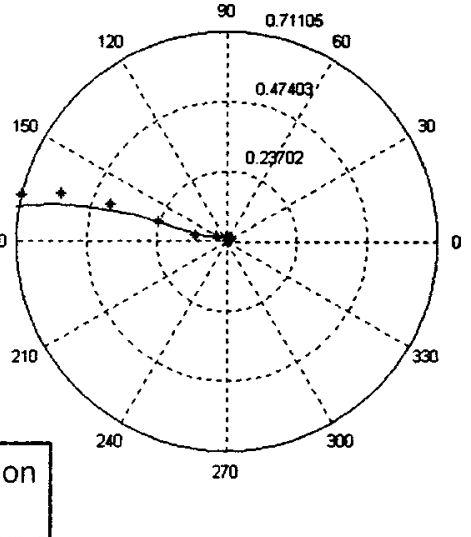

(b)

Fig. 3. Output envelopes of: (a) carrier and (b) $\mathrm{IM}_{3}$.

the resulting baseband equivalent output signal is

$$
\begin{aligned}
y_{o}(t)= & \sum_{k=1}^{n} f_{2 k-1} x_{o}^{2 k-1}(t) \\
= & \sum_{k=1}^{n} f_{2 k-1} s^{2 k-1} \cos ^{2 k-1}(\Delta \omega t) \\
= & f_{1} s \cos (\Delta \omega t)+f_{3} s^{3} \cos ^{3}(\Delta \omega t)+\cdots \\
& +f_{2 n-1} s^{2 n-1} \cos ^{2 n-1}(\Delta \omega t) \\
= & \left(f_{1} s+\frac{3}{4} f_{3} s^{3}+\cdots+\frac{2 n-1}{4^{n-1}} f_{2 n-1} s^{2 n-1}\right) \\
& \cdot \cos (\Delta \omega t)+\left(\frac{1}{4} f_{3} s^{3}+\cdots+\frac{2 n-1}{4^{n-1}} f_{2 n-1} s^{2 n-1}\right) \\
& \cdot \cos (3 \Delta \omega t)+\cdots+\left(\frac{1}{4^{n-1}} f_{2 n-1} s^{2 n-1}\right) \\
& \cdot \cos ((2 n-1) \Delta \omega t) \\
= & y_{1} \cos (\Delta \omega t)+y_{3} \cos (3 \Delta \omega t)+\cdots \\
& +y_{2 n-1} \cos ((2 n-1) \Delta \omega t) \\
= & \sum_{k=1}^{n} y_{2 k-1} \cos ((2 k-1) \Delta \omega t) \\
&
\end{aligned}
$$

where $y_{2 k-1}$ is an output complex envelope of $\mathrm{IM}_{2 k-1}$. The general expression of $y_{2 k-1}$ is

$$
y_{2 k-1}=\sum_{j=k}^{n} \frac{2 j-1}{4^{j-1}} f_{2 j-1} s^{2 j-1}
$$

and it relates to the $\mathrm{IM}_{2 k-1}$ output power as

$$
P_{\mathrm{IM}, 2 k-1}=\frac{1}{2}\left|\frac{y_{2 k-1}}{2}\right|^{2} \frac{1}{R}=\frac{\left|y_{2 k-1}\right|^{2}}{8 R}(W)
$$

where $P_{\mathrm{IM} 1}$ is carrier frequency output power, and $P_{\mathrm{IM}, 2 k-1}$ $(k=2,3,4, \ldots)$ are $(2 k-1)$ th order IM output power. Equations (11) and (12) show that, if a power amplifier is properly modeled, i.e., if coefficients $f_{2 k-1}$ are correctly determined, we

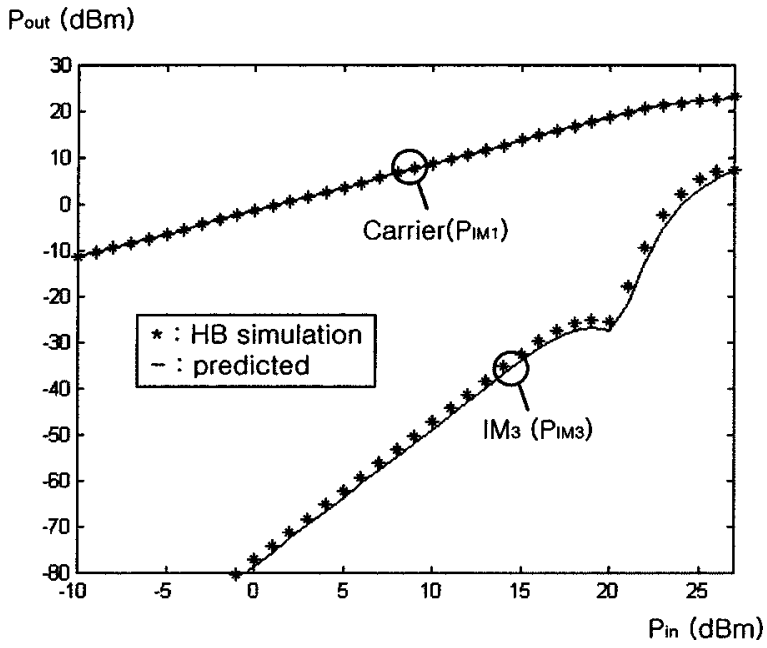

Fig. 4. Output power characteristics of carrier and $\mathrm{IM}_{3}$.

can calculate the general-order IM powers as a function of input amplitude $s$.

To verify our analysis, we compare the output envelopes predicted by (11) with those by harmonic-balance (HB) simulation, which are performed on the commercial computer-aided design (CAD) tool HP Microwave Design System (MDS). Using MDS, we design a 2-GHz band power amplifier, perform a two-tone test $(1.99975 \mathrm{GHz}, 2.00025 \mathrm{GHz})$, and finally obtain the output envelopes of carrier and $\mathrm{IM}_{3}$. At the same time, the output envelopes are predicted by (11) with the mathematical model of the power amplifier. The coefficients $f_{2 k-1}$ are determined by the odd-order polynomial fitting of the complex envelope transfer function, which is obtained by AM-AM and AM-PM distortions of a carrier one-tone. The two output envelopes are then plotted together, as shown in Fig. 3, to compare the results. Since the output envelopes have complex values, they are displayed in polar form. As can be seen in Fig. 3, the two output envelopes are much close to each other for both carrier and $\mathrm{IM}_{3}$. It shows that both the magnitude and phase of each frequency component are predicted well by (11). In Fig. 3, the agreement in the small-signal region is hard to see because of the small-signal amplitude. Therefore, we present the results in another way, as shown in Fig. 4, 
where the power characteristics of carrier and $\mathrm{IM}_{3}$ are shown. Excellent agreement between the two results is obtained, from the small- to large-signal regions. Though we did not show the phase characteristic, it also gives good agreement between the two results. From Figs. 3 and 4, we verify the mathematical model of a power amplifier and the analytical expressions of IM components. Though we did not compare it with the real measurement results, HB simulation is known as the most accurate simulation method for a real system, and we conclude that Figs. 3 and 4 verify our analysis.

\section{ANALYTICAL EXPRESSION OF OUtPUT SPECTRUM IN CDMA SIGNAL INPUT}

The derivation presented in this section is similar to that of Wu [5]-[7]. The most different thing is that while Wu use an AM-AM distortion only, we use a complex envelope transfer function as a nonlinear power amplifier model in order to explain the effects of AM-PM distortion on the amplified CDMA output spectrum. A general input of the QPSK modulated CDMA signal is

$$
\begin{aligned}
x(t) & =I(t) \cos \omega_{c} t-Q(t) \sin \omega_{c} t \\
& =\sqrt{I^{2}(t)+Q^{2}(t)} \cos \left(\omega_{c} t+\tan ^{-1} \frac{Q(t)}{I(t)}\right)
\end{aligned}
$$

where $I(t)$ and $Q(t)$ are the in-phase and quadrature components of a baseband signal, respectively. The baseband equivalent input signal is then

$$
x_{o}(t)=\sqrt{I^{2}(t)+Q^{2}(t)}
$$

which is a bandlimited white Gaussian process. An output spectrum can be calculated using a power amplifier's baseband equivalent model since the power spectrum of $y(t)$ and that of $y_{o}(t)$ have the following relation, which is derived from (2):

$$
P_{y}(f)=\frac{1}{4 R}\left\{P_{y_{o}}\left(f-f_{c}\right)+P_{y_{o}}\left(f+f_{c}\right)\right\}
$$

Therefore, in order to calculate the output spectrum $P_{y}(f)$, we only calculate the baseband output spectrum $P_{y o}(f)$ and shift this spectrum to carrier frequency $f_{c}$. By definition, a power spectrum is equal to a Fourier transformed autocorrelation function, and $P_{y o}(f)$ can be obtained from its autocorrelation function $R_{y o}(\tau)$ as

$$
P_{y o}(f)=F\left[R_{y o}(\tau)\right]=\int_{-\infty}^{\infty} R_{y o}(\tau) e^{-j \omega \tau} d \tau
$$

where $F[\cdot]$ denotes the Fourier transform of [.]. Also by definition, $R_{y o}(\tau)$ is expressed as

$$
R_{y o}(\tau)=E\left[y_{o}(t) y_{o}^{*}(t+\tau)\right]
$$

where $E[\cdot]$ denotes the mathematical expectation of [.]. Putting (6) into (17), and recalling that $x_{o}(t)$ is a real signal

$$
\begin{aligned}
R_{y o}(\tau) & =E\left[\left(\sum_{j=1}^{n} f_{2 j-1} x_{o}^{2 j-1}(t)\right)\left(\sum_{k=1}^{n} f_{2 k-1} x_{o}^{2 k-1}(t+\tau)\right)^{*}\right] \\
& =\sum_{j=1}^{n} \sum_{k=1}^{n} f_{2 j-1} f_{2 k-1}^{*} E\left[x_{o}^{2 j-1}(t) x_{o}^{2 k-1}(t+\tau)\right] .
\end{aligned}
$$

The calculation of the expectation $E[\cdot]$ in summation is quite involved. To develop the expectation, we use Isserlis's formula [10]

$$
\begin{aligned}
& E\left[X_{1} X_{2} \cdots X_{r-1} X_{r}\right] \\
& \quad=E\left[X_{1} X_{2}\right] E\left[X_{3} X_{4}\right] \cdots E\left[X_{r-1} X_{r}\right] \\
& \quad+E\left[X_{1} X_{3}\right] E\left[X_{2} X_{4}\right] \cdots E\left[X_{r-1} X_{r}\right]+\cdots
\end{aligned}
$$

where $r$ is even integer and $X_{i}$ are zero mean Gaussian variables. The summation is taken over all possible ways of dividing the $r$ integers into $r / 2$ combinations of pairs. For example, for $r=4$

$$
\begin{array}{r}
E\left[X_{1} X_{2} X_{3} X_{4}\right]=E\left[X_{1} X_{2}\right] E\left[X_{3} X_{4}\right]+E\left[X_{1} X_{3}\right] E\left[X_{2} X_{4}\right] \\
+E\left[X_{1} X_{4}\right] E\left[X_{2} X_{3}\right] .
\end{array}
$$

Using this Isserlis's formula and applying a tedious manipulation, we obtain

$$
\begin{aligned}
R_{y o}(\tau)= & \frac{1}{1 !} \mid \frac{1 !}{2^{0} \cdot 0 !} f_{1}+\frac{3 !}{2^{1} \cdot 1 !} f_{3} K+\frac{5 !}{2^{2} \cdot 2 !} f_{5} K^{2}+\cdots \\
& +\left.\frac{(2 n-1) !}{2^{n-1} \cdot(n-1) !} f_{2 n-1} K^{n-1}\right|^{2} R_{x o}(\tau) \\
+ & \frac{1}{3 !} \mid \frac{3 !}{2^{0} \cdot 0 !} f_{3}+\frac{5 !}{2^{1} \cdot 1 !} f_{5} K+\frac{7 !}{2^{2} \cdot 2 !} f_{7} K^{2}+\cdots \\
& \quad+\left.\frac{(2 n-1) !}{2^{n-2} \cdot(n-2) !} f_{2 n-1} K^{n-2}\right|^{2} R_{x o}^{3}(\tau) \\
& +\cdots+\frac{1}{(2 k-1) !} \\
& \cdot \mid \frac{(2 k-1) !}{2^{0} \cdot 0 !} f_{2 k-1}+\frac{(2 k+1) !}{2^{1} \cdot 1 !} f_{2 k+1} K+\cdots \\
& +\left.\frac{(2 n-1) !}{2^{n-k} \cdot(n-k) !} f_{2 n-1} K^{n-k}\right|^{2} R_{x o}^{2 k-1}(\tau)+\cdots \\
& +\frac{1}{(2 n-3) !}\left|\frac{(2 n-3) !}{2^{0} \cdot 0 !} f_{2 n-3}+\frac{(2 n-1) !}{2^{1} \cdot 1 !} f_{2 n-1} K\right|^{2} \\
& \cdot R_{x o}^{2 n-3}(\tau)+\frac{1}{(2 n-1) !}\left|\frac{(2 n-1) !}{2^{0} \cdot 0 !} f_{2 n-1}\right|^{2} R_{x o}^{2 n-1}(\tau)
\end{aligned}
$$

where

$$
\begin{aligned}
R_{x o}(\tau) & =E\left[x_{o}(t) x_{o}(t+\tau)\right] \\
K & =R_{x o}(0)=E\left[x_{o}^{2}(t)\right] .
\end{aligned}
$$


The above expression of $R_{y o}(\tau)$ seems to be very complicated, but can be simplified as

$$
R_{y o}(\tau)=\sum_{k=1}^{n} a_{2 k-1} R_{x o}^{2 k-1}(\tau)
$$

where

$$
a_{2 k-1}=\frac{1}{(2 k-1) !}\left|\sum_{j=0}^{n-k} \frac{(2 j+2 k-1) !}{2^{j} \cdot j !} f_{2 j+2 k-1} K^{j}\right|^{2} .
$$

Using (16), we can derive an expression for the power spectrum $P_{y o}(f)$ as

$$
\begin{aligned}
P_{y o}(f) & =F\left[R_{y o}(\tau)\right] \\
& =\sum_{k=1}^{n} a_{2 k-1} F\left[R_{x o}^{2 k-1}(\tau)\right] \\
& =\sum_{k=1}^{n} a_{2 k-1} P_{2 k-1}(f)
\end{aligned}
$$

where

$$
\begin{gathered}
P_{2 k-1}(f)=\underbrace{P_{x o}(f) \otimes P_{x o}(f) \otimes \cdots \otimes P_{x o}(f)}_{2 k-1 \text { times }}, \\
\otimes: \quad \text { convolution. }
\end{gathered}
$$

Equation (25) shows that an input spectrum is directly relates to an output spectrum. That is, if a power amplifier is properly modeled, an output spectrum can be calculated by an input spectrum with some convolution processes.

Now, we will derive an explicit expression of an output spectrum assuming a rectangular pulse-shaping filter (PSF). Though a raised-root cosine (RRC) filter is used in many communication systems, its spectrum is quite similar to that of a rectangular filter, while its convolution is much more complicated, and, therefore, we will derive an analytical expression of a CDMA output spectrum only for a rectangular filter. When a rectangular PSF is used, a power spectral density (PSD) of the baseband equivalent input signal $x_{o}(t)$ is

$$
P_{x o}(f)=\left\{\begin{array}{l}
N_{o} / 2, \quad|f| \leq B \\
0, \quad|f|>B
\end{array}\right.
$$

where $B$ is the bandwidth of the PSF. The autocorrelation function of this input signal is

$$
\begin{gathered}
R_{x o}(\tau)=E\left[x_{o}(t) x_{o}(t+\tau)\right]=\frac{N_{o} \sin (2 \pi B \tau)}{2 \pi \tau} \\
K=R_{x o}(0)=E\left[x_{o}^{2}(t)\right]=N_{o} B
\end{gathered}
$$

and the total input power is

$$
P_{i}=\frac{1}{2}\left(\frac{N_{o}}{2} \times 2 B\right) \frac{1}{R}=\frac{N_{o} B}{2 R} .
$$

To obtain $P_{2 k-1}(f)$, convolution is performed on the input spectrum in (27). Since $P_{x o}(f)$ has a rectangular shape, the calculation of its convolution is straightforward, and we get the equations shown at the bottom of this page. In general,

$$
P_{2 k-1}(f)=\left\{\begin{array}{c}
\frac{1}{(2 k-2) !} \frac{1}{B}\left(\frac{K}{2}\right)^{2 k-1} \\
\cdot\left[\sum_{r=0}^{k-q}(-1)^{r}{ }_{2 k-1} C_{r}\left((2 k-2 r-1)-\frac{|f|}{B}\right)^{2 k-2}\right] \\
\text { for }(2 q-3) B \leq|f|<(2 q-1) B, \\
1 \leq q \leq k \\
0, \quad \text { for }|f|>(2 k-1) B
\end{array}\right.
$$

where $N_{o} B$ is replaced by $K$. Note that $P_{2 k-1}(f)$ is different for each frequency band since the different order of convolution is performed on the bandlimited input spectrum $P_{x o}(f)$. We have now obtained $a_{2 k-1}$ and $P_{2 k-1}(f)$ in (24) and (30),

$$
\begin{aligned}
& P_{3}(f)= \begin{cases}\frac{1}{2 !} \frac{1}{B}\left(\frac{N_{o} B}{2}\right)^{3}\left[{ }_{3} C_{0}\left(3-\frac{|f|}{B}\right)^{2}-{ }_{3} C_{1}\left(1-\frac{|f|}{B}\right)^{2}\right], & \text { for }|f|<B \\
\frac{1}{2 !} \frac{1}{B}\left(\frac{N_{o} B}{2}\right)^{3}\left[{ }_{3} C_{0}\left(3-\frac{|f|}{B}\right)^{2}\right], \quad \text { for } B \leq|f|<3 B & \\
0, \quad \text { for }|f| \geq 3 B & \end{cases} \\
& P_{5}(f)=\left\{\begin{array}{lll}
\frac{1}{4 !} \frac{1}{B}\left(\frac{N_{o} B}{2}\right)^{5}\left[{ }_{5} C_{0}\left(5-\frac{|f|}{B}\right)^{4}-{ }_{5} C_{1}\left(3-\frac{|f|}{B}\right)^{4}+{ }_{5} C_{2}\left(1-\frac{|f|}{B}\right)^{4}\right], & \text { for }|f|<B \\
\frac{1}{4 !} \frac{1}{B}\left(\frac{N_{o} B}{2}\right)^{5}\left[{ }_{5} C_{0}\left(5-\frac{|f|}{B}\right)^{4}-{ }_{5} C_{1}\left(3-\frac{|f|}{B}\right)^{4}\right], & \text { for } B \leq|f|<3 B \\
\frac{1}{4 !} \frac{1}{B}\left(\frac{N_{o} B}{2}\right)^{5}\left[{ }_{5} C_{0}\left(5-\frac{|f|}{B}\right)^{4}\right], & \text { for } 3 B \leq|f|<5 B & \\
0, & \text { for }|f| \geq 5 B
\end{array}\right.
\end{aligned}
$$


respectively. We can derive a complete expression of $P_{y o}(f)$ from (25)

$$
\begin{aligned}
P_{y o}(f) & \sum_{k=1}^{n} a_{2 k-1} P_{2 k-1}(f) \\
= & \sum_{k=1}^{n}\left[\left(\frac{1}{(2 k-1) !}\left|\sum_{j=0}^{n-k} \frac{(2 j+2 k-1) !}{2^{j} \cdot j !} f_{2 j+2 k-1} K^{j}\right|^{2}\right)\right. \\
& \cdot\left(\frac{1}{(2 k-2) !} \frac{1}{B}\left(\frac{K}{2}\right)^{2 k-1}\right. \\
& \left.\left.\cdot\left\{\sum_{r=0}^{k-q}(-1)^{r}{ }_{2 k-1} C_{r}\left((2 k-2 r-1)-\frac{|f|}{B}\right)^{2 k-2}\right\}\right)\right],
\end{aligned}
$$$$
\text { for }(2 q-3) B \leq|f|<(2 q-1) B, \quad 1 \leq q \leq k \text {. }
$$

In (31), the main channel is $|f|<B(q=1)$, the adjacent channel is $B<|f|<3 B(q=2)$, and the alternative channel is $3 B<|f|<5 B(q=3)$. Equation (31) shows that if $f_{2 k-1}$ are correctly determined and an input spectrum is given, the baseband output spectrum $P_{y o}(f)$ can be theoretically calculated up to frequency band $(2 n-1) B$. It also shows that each $(2 k-1)$ th-order distortion characterized by $f_{2 k-1}$ affects the spectrum regrowth in the band $|f|<(2 k-1) B$. The output spectrum in the band $|f|>(2 n-1) B$ is zero, which is a consequence of assuming a bandlimited input spectrum. In practice, there is no bandlimited input signal, and the output spectrum is not zero in the band $|f|>(2 n-1) B$. Usually, the thirdand fifth-order distortions are dominant, and (31) can be simplified for the case of $n=3$, as shown in (32), at the bottom of this page. To get an output spectrum $P_{y}(f), P_{y o}(f)$ should be scaled and shifted, as in (15). Using this result, we can predict the amplified output spectrum of the CDMA signal.

\section{RELATIONSHIP BETWEEN IM PRODUCTS AND CDMA OUTPUT SPECTRUM}

Using the expressions previously derived, we can relate the IM products to the CDMA output spectrum. When a power amplifier is operated in a weakly nonlinear region, it is a reasonable assumption that the amplifier has only third- and fifth-order distortions. Then, $n=3$, and the coefficients $y_{2 k-1}$ in (11) will be

$$
\begin{aligned}
& y_{1}=f_{1} s+\frac{3}{4} f_{3} s^{3}+\frac{5}{8} f_{5} s^{5} \\
& y_{3}=\frac{1}{4} f_{3} s^{3}+\frac{5}{16} f_{5} s^{5} \\
& y_{5}=\frac{1}{16} f_{5} s^{5} .
\end{aligned}
$$

Note that each phase of $y_{2 k-1}$ has a different value since each phase of $f_{2 k-1}$ has a different value. Solving (33) for $f_{2 k-1}$ yields

$$
\begin{aligned}
f_{1} & =\frac{1}{s}\left(y_{1}-3 y_{3}+5 y_{5}\right) \\
& =\frac{1}{s}\left(\left|y_{1}\right| e^{j \theta_{y 1}}-3\left|y_{3}\right| e^{j \theta_{y 3}}+5\left|y_{5}\right| e^{j \theta_{y 5}}\right) \\
f_{3} & =\frac{4}{s^{3}}\left(y_{3}-5 y_{5}\right) \\
& =\frac{4}{s^{3}}\left(\left|y_{3}\right| e^{j \theta_{y 3}}-5\left|y_{5}\right| e^{j \theta_{y 5}}\right) \\
f_{5} & =\frac{16}{s^{5}}\left(y_{5}\right) \\
& =\frac{16}{s^{5}}\left(\left|y_{5}\right| e^{j \theta_{y 5}}\right)
\end{aligned}
$$

where each complex output envelope is decomposed into its magnitude and phase terms. Using (12), the magnitude term can be obtained by its IM power as

$$
\left|y_{2 k-1}\right|=\sqrt{8 R P_{\mathrm{IM}, 2 k-1}} \text {. }
$$

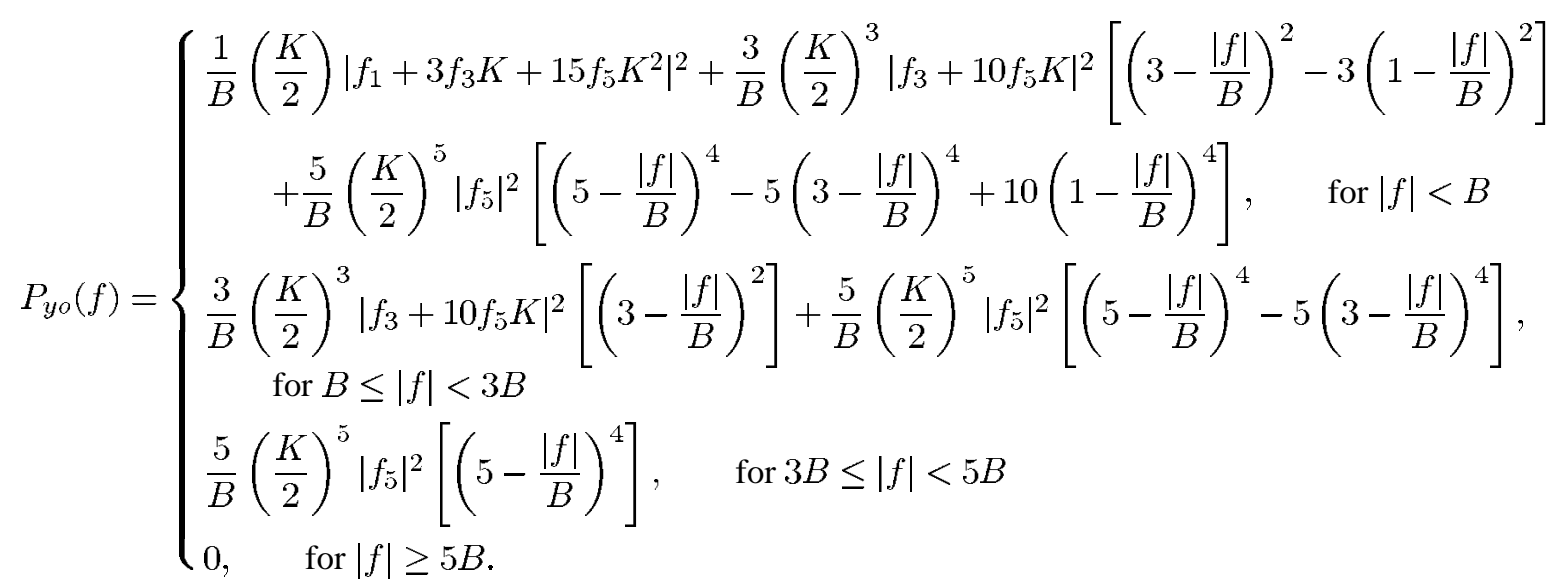


For the same power in a continuous-wave (CW) two-tone and CDMA signal input, $P_{i}$ in (8) and (29) should be equal as follows:

$$
P_{i}=\frac{s^{2}}{4 R}=\frac{N_{o} B}{2 R}, \quad \therefore N_{o} B=K=\frac{s^{2}}{2} .
$$

Applying (34)-(36) to (32) yields the baseband output spectrum $P_{y o}(f)$ and then using (15), we can derive an analytical expression of the CDMA output spectrum $P_{y}(f)$, as shown in (37), at the bottom of this page. This equation directly relates the IM products in the two-tone input to the output spectrum in the CDMA signal input. If the IM powers and phases of a two-tone test are measured, the output spectrum of a CDMA signal can be predicted with a given bandwidth $B$. To show the effects of AM-PM distortion on the CDMA output spectrum, we calculate (37) in the following four special cases:

Case 1) $\theta_{y 1}=\theta_{y 3}=\theta_{y \tilde{5}}$;

Case 2) $\theta_{y 1}=\pi+\theta_{y 3}=\theta_{y 5}$;

Case 3) $\theta_{y 1}=\theta_{y 3}=\pi+\theta_{y \check{5}}$;

Case 4) $\theta_{y 1}=\pi+\theta_{y 3}=\pi+\theta_{y 5}$.

The result is shown in Fig. 5, where the spectrum predicted by $\mathrm{IM}_{3}$ only $\left[P_{\mathrm{IM}}=0\right.$ in (37) $]$ is also plotted for comparison. The alternative channel $3 B<\left|f-f_{c}\right|<5 B$ is not presented since all four spectrums are equal in this channel. Fig. 5 clearly shows that the CDMA output spectrum varies as the IM phase variations, which means that AM-PM distortion also affects the CDMA output spectrum. Note that the $\mathrm{IM}_{5}$ phase relative to the $\mathrm{IM}_{3}$ phase has dominant effects on an adjacent channel, and the $\mathrm{IM}_{3}$ phase relative to the $\mathrm{IM}_{1}$ phase on the main channel. Thus far, as the ACP is concerned, we can state that case 3 is the best and case 4 is the worst. Though we cannot measure the accurate IM phases, cases 3 and 4 give the upper and lower limit of the output spectrum, respectively, and, using this, we can estimate the CDMA output spectrum. The difference between the two limits will be reduced if $P_{\mathrm{IM} 3}$ is much smaller than $P_{\mathrm{IM} 1}$ and $P_{\mathrm{IM} 5}$ is much smaller than $P_{\mathrm{IM} 3}$. Generally, the real CDMA

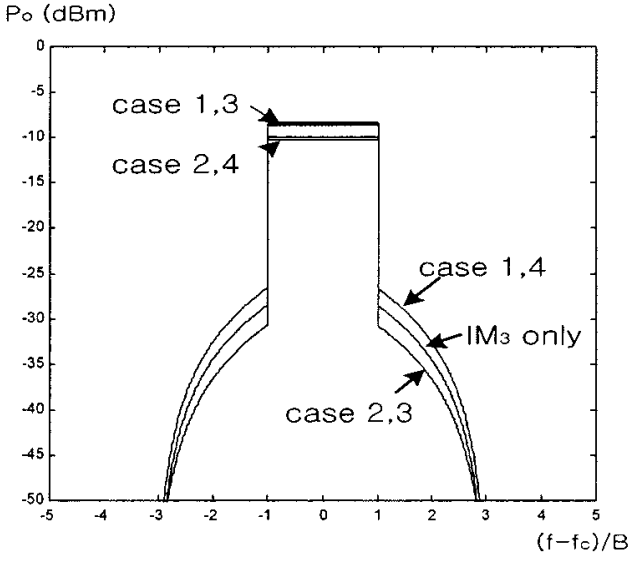

Fig. 5. Effects of IM phase variation on the CDMA output spectrum. Case 1: $\theta_{y 1}=\theta_{y 3}=\theta_{y 5}$. Case 2: $\theta_{y 1}=\pi+\theta_{y 3}=\theta_{y 5}$. Case 3: $\theta_{y 1}=\theta_{y 3}=$ $\pi+\theta_{y 5}$. Case 4: $\theta_{y 1}=\pi+\theta_{y 3}=\pi+\Theta_{y 5}$.

output spectrum lies between the two limits, and, for this point, (37) would be useful to predict the CDMA output spectrum.

\section{COMPARISON BETWEen THE MEASURED AND PREDICTED RESULTS}

To verify our derivation, we perform a real measurement with an RF power amplifier designed for a WCDMA system. The carrier frequency is $1967.5 \mathrm{MHz}$ and the signal bandwidth is $4.096 \mathrm{MHz}$ ( $B=2.048 \mathrm{MHz}$ ). To predict the CDMA output spectrum, we first measure the IM powers from a two-tone test with two 1966.5- and 1968.5-MHz carriers. For the input power of $-12 \mathrm{dBm}$, the measured $P_{\mathrm{IM} 1}, P_{\mathrm{IM} 3}$, and $P_{\mathrm{IM} 5}$ are 9.0, -20.2 , and $-55.8 \mathrm{dBm}$ for the lower sideband, and 8.9, -19.9 , and $-56.2 \mathrm{dBm}$ for the upper sideband, respectively. It should be noted that since, in our derivations, the $f_{2 k-1}$ terms are obtained by a carrier one-tone measurement based on the narrow-band assumption, IM and ACP are all symmetric around the carrier frequency. In a real situation, however, they

$$
P_{y}(f)=\left\{\begin{array}{c}
\frac{1}{2 B}\left|\sqrt{P_{\mathrm{IM} 1}} e^{j \theta_{y 1}}+3 \sqrt{P_{\mathrm{IM} 3}} e^{j \theta_{y 3}}+35 \sqrt{P_{\mathrm{IM} 5}} e^{j \theta_{y 5}}\right|^{2}+\frac{3}{2 B}\left|\sqrt{P_{\mathrm{IM} 3}} e^{j \theta_{y 3}}+15 \sqrt{P_{\mathrm{IM} 5}} e^{j \theta_{y 5}}\right|^{2} \\
\cdot\left[\left(3-\frac{\left|f-f_{c}\right|}{B}\right)^{2}-3\left(1-\frac{\left|f-f_{c}\right|}{B}\right)^{2}\right]+\frac{5}{2 B} \mid \sqrt{P_{\mathrm{IM} 5}} e^{\left.j \theta_{y 5}\right|^{2}} \\
\cdot\left[\left(5-\frac{\left|f-f_{c}\right|}{B}\right)^{4}-5\left(3-\frac{\left|f-f_{c}\right|}{B}\right)^{4}+10\left(1-\frac{\left|f-f_{c}\right|}{B}\right)^{4}\right], \quad \text { for }\left|f-f_{c}\right|<B \\
\frac{3}{2 B}\left|\sqrt{P_{\mathrm{IM} 3}} e^{j \theta_{y 3}}+15 \sqrt{P_{\mathrm{IM} 5}} e^{j \theta_{y 5} \mid}\right|^{2}\left[\left(3-\frac{\left|f-f_{c}\right|}{B}\right)^{2}\right]+\frac{5}{2 B}\left|\sqrt{P_{\mathrm{IM} 5}} e^{j \theta_{y 5}}\right|^{2} \\
\cdot\left[\left(5-\frac{\left|f-f_{c}\right|}{B}\right)^{4}-5\left(3-\frac{\left|f-f_{c}\right|}{B}\right)^{4}\right], \quad \text { for } B \leq\left|f-f_{c}\right|<3 B \\
\frac{5}{2 B}\left|\sqrt{P_{\mathrm{IM} 5}} e^{j \theta_{y 5}}\right|^{2}\left[\left(5-\frac{\left|f-f_{c}\right|}{B}\right)^{4}\right], \quad \text { for } 3 B \leq\left|f-f_{c}\right|<5 B \\
0, \quad \text { for }\left|f-f_{c}\right| \geq 5 B .
\end{array}\right.
$$




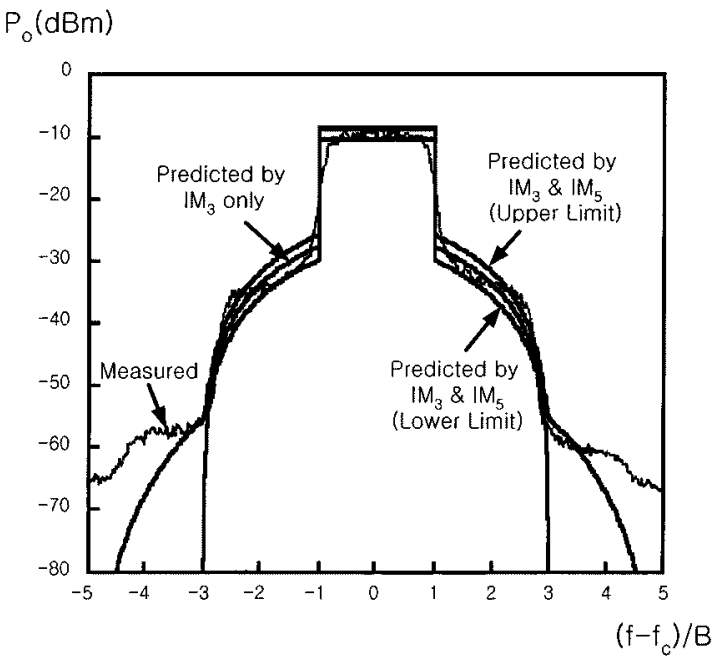

Fig. 6. Measured and predicted output spectrums $\left(f_{c}=1.9675 \mathrm{GHz}, B=\right.$ $2.048 \mathrm{MHz}$.

do exhibit asymmetry due to the frequency dependence of the power amplifier, which is explained in [9] and [11]. In our study, the difference between the lower and upper IM is not much, and the frequency dependence is ignored. However, when the difference is large, the frequency dependence should be included in a power amplifier's model, which will be left for future works. To continue our current study, IM powers of lower and upper sidebands are averaged as

$$
P_{\mathrm{IM}, 2 k-1}=10 \log \left[\left(10^{\Gamma_{\mathrm{IM} L, 2 k-1} / 10}+10^{\Gamma_{\mathrm{IM} H, 2 k-1} / 10}\right) / 2\right]
$$

and the IM powers $P_{\mathrm{IM} 1}, P_{\mathrm{IM} 3}$, and $P_{\mathrm{IM} s}$ are determined to be $9,-20$, and $-56 \mathrm{dBm}$, respectively. From these IM powers, the CDMA output spectrum of an equal input power is then calculated using (37). Next, we measure the real output spectrum of a CDMA signal. The real CDMA signal is generated by an SMIQ03 signal generator, where the RRC filter with a rolloff factor of 0.22 is used for pulse shaping. The output spectrum is measured by an HP 8594E spectrum analyzer with the resolution bandwidth (RBW) of $30 \mathrm{kHz}$. To compare the measured and predicted results, they are plotted together in Fig. 6. In this figure, the predicted output spectrums are converted to the analyzer's RBW, which means that the predicted spectrums are multiplied by the RBW.

Several observations can be made from Fig. 6. In the band $\left|f-f_{c}\right|<3 B$, the measured spectrum lies between the upper and lower limits of the predicted spectrum. Though an accurate output spectrum cannot be predicted, its variation range can be predicted by the two limits, and this helps to estimate the CDMA output spectrum. The spectrum predicted by $\mathrm{IM}_{3}$ only also gives good results, for it lies in the middle of the upper and lower limits in the adjacent channel. Generally, the more accurate output spectrum can be obtained if the more higher order IM products are considered. However, if the fifth-order or higher order IM power is $30 \mathrm{~dB}$ or more lower than the third-order IM power, the output spectrum can be predicted well by $\mathrm{IM}_{3}$ only.
The difference in the band $\left|f-f_{c}\right|>3 B$ is due to the fact that a real system has a finite noise level. This finite noise level flattens out the output spectrum in this band. If the noise level is much lower than that in Fig. 6, the predicted spectrum will agree with the measured spectrum, which is also in the band $\left|f-f_{c}\right|>3 B$. With all the errors resulting from a finite noise level and ignored higher order IM products, the real output spectrum can be predicted well by (37), which verifies our derivations.

\section{CONCLUSION}

In this paper, we have derived an analytical expression that relates IM products in a $\mathrm{CW}$ two-tone input to an output spectrum in a CDMA signal input. From this expression, we show that, not only IM powers, but also IM phases, affect the CDMA output spectrum. In other words, not only AM-AM, but also AM-PM distortion affects the CDMA output spectrum. The $\mathrm{IM}_{5}$ phase relative to the $\mathrm{IM}_{3}$ phase especially has dominant effects on an adjacent channel, and the $\mathrm{IM}_{3}$ phase relative to the $\mathrm{IM}_{1}$ phase on a main channel. Since it is difficult to measure the accurate IM phases, it is also difficult to predict the accurate CDMA output spectrum, and, as an alternative, we present the upper and lower limits of output spectrum. The real measurement shows that the measured output spectrum lies between these two limits of the predicted spectrum, which verifies our work. The difference between the two limits will be reduced if $P_{\mathrm{IM} 3}$ is much smaller than $P_{\mathrm{IM} 1}$ and $P_{\mathrm{IM} \tilde{}}$ is much smaller than $P_{\mathrm{IM} 3}$. The spectrum predicted by $\mathrm{IM}_{3}$ only also gives good results since it lies in the middle of the two limits.

In our derivation, an RF power amplifier is mathematically modeled by a complex envelope transfer function not decomposing it into AM-AM and AM-PM distortions. The reason is that if a complex envelope transfer function is decomposed, the effect of AM-PM distortion is hard to analyze, as shown in Section II. Since AM-PM distortion has amplitude quantity, as well as phase quantity, it should be considered in predicting the IM and CDMA output spectrum and, for this purpose, we use a complex envelope transfer function. For verification, we derive an analytical expression of IM products of a two-tone test, and compare it with the HB simulation results. Excellent agreement is obtained between the two results, both for the power and phase of $\mathrm{IM}_{1}$ and $\mathrm{IM}_{3}$.

All the expressions in this paper are derived in general forms, and the IM products or CDMA output spectrum can be predicted in any order. For example, $\mathrm{IM}_{7}$ or more higher order IM products can be predicted. Therefore, this paper will help RF system designers to predict the distortion effects of an RF power amplifier, and it is thought to be useful for future software radio applications.

\section{REFERENCES}

[1] A. R. Kaye, D. A. George, and M. J. Eric, "Analysis and compensation of bandpass nonlinearities for communications," IEEE Trans. Commun., vol. 21, pp. 965-972, Oct. 1972.

[2] S. W. Chen, W. Panton, and R. Gilmore, "Effects of nonlinear distortion on CDMA communication systems," IEEE Trans. Microwave Theory Tech., vol. 44, pp. 2743-2749, Dec. 1996.

[3] J. F. Sevic and J. Staudinger, "Simulation of power amplifier adjacentchannel power ratio for digital wireless communication systems," in Proc. 47th IEEE Veh. Technol. Conf., 1997, pp. 681-685. 
[4] V. Borich, J. H. Jong, J. East, and W. E. Stark, "Nonlinear effects of power amplification on multicarrier spread spectrum systems," in IEEE MTT-S Int. Microwave Symp. Dig., 1998, pp. 323-326.

[5] Q. Wu, M. Testa, and R. Larkin, "Linear RF power amplifier design for CDMA signals," in IEEE MTT-S Int. Microwave Symp. Dig., 1996, pp. $851-854$.

[6] — "On design of linear RF power amplifier for CDMA signals," Int. J. Microwave Millimeter-Wave Computer-Aided Eng., pp. 283-292, Feb. 1998.

[7] Q. Wu, H. Xiao, and F. Li, "Linear RF power amplifier design for CDMA signals : A spectrum analysis approach," Microwave J., pp. 22-40, Dec. 1998.

[8] N. Suematsu, Y. Iyama, and O. Ishida, "Transfer characteristic of $\mathrm{IM}_{3}$ relative phase for a GaAs FET amplifier," IEEE Trans. Microwave Theory Tech., vol. 45, pp. 2509-2514, Dec. 1997.

[9] J. F. Sevic, K. L. Burger, and M. B. Steer, "A novel envelope-termination load-pull method for ACPR optimization of RF/microwave power amplifiers," in IEEE MTT-S Int. Microwave Symp. Dig., 1998, pp. 723-726.

[10] W. A. Gardner, Introduction to Random Processes, 2nd ed. New York: McGraw-Hill, 1990.

[11] K. Horiguchi, K. Yamauchi, K. Mori, M. Nakayama, and T. Takagi, "A novel distortion analysis method for amplifiers considering frequency characteristics," in IEEE MTT-S Int. Microwave Symp. Dig., 1998, pp. $1619-1622$

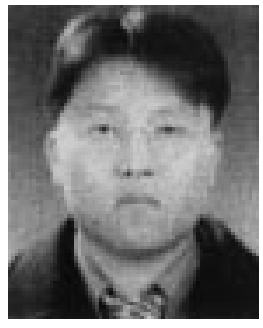

Seung-June Yi was born in Seoul, Korea, in 1972. He received the B.S. and M.S. degrees in electronics engineering from the Seoul National University, Seoul, Korea, in 1994 and 1996, respectively, and is currently working toward the Ph.D. degree at the Seoul National University.

Since 1999, he has also been an Associate Research Engineer at LG Electronics Inc., Anyang, Korea, where he has been with Advanced Telecommunication Research Laboratory, and where he is involved with IMT-2000 (WCDMA) standardization. His interests include design and computer simulation of RF systems in wireless communications.

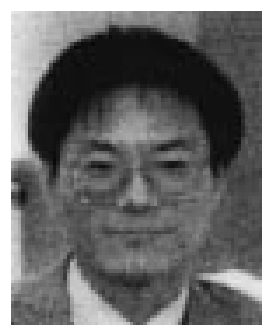

Sangwook Nam (S'87-M'88) received the B.S. degree from the Seoul National University, Seoul, Korea, in 1981, the M.S. degree from the Korea Advanced Institute of Science and Technology, Seoul, Korea, in 1983, and the Ph.D. degree from The University of Texas at Austin, in 1989, all in electrical engineering

From 1983 to 1986 , he was a Researcher at the GoldStar Central Research Laboratory, Seoul, Korea. Since 1990, he has been with the Seoul National University, where he is currently a Professor in the School of Electrical Engineering. His research interests include analysis/design of electromagnetic structures, antennas, and microwave active/passive circuits.

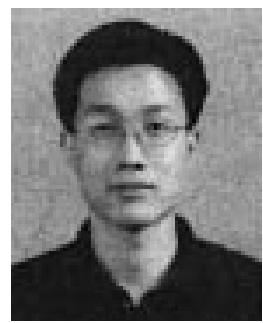

Sung-Hoon Oh was born in Kyung-Sang Do, Korea, in 1968. He received the M.S. degree in electronics engineering from the Inha University, $<$ City, $>$ Korea, in 1995 .

From 1995 to 1997, he was an Assistant Research Engineer, during which time he developed a generalized scattering matrix (GSM) terminal at the $\mathrm{N}-\mathrm{Hi}$ Center, LG Electronics Inc. Since 1998, he has been with the Next Generation Communication Laboratory, LG Electronics Inc., Kyongki-do, Korea, where he is currently he is a Research Engineer, involved with the research and development of the IMT-2000 (WCDMA) terminal. His main interests are RF circuit design and electromagnetic wave propagation.

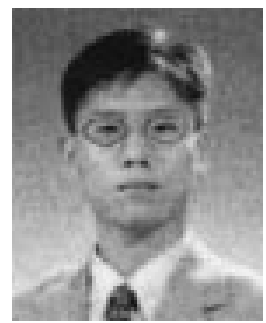

Jae-Hee Han (S'00) was born in Seoul, Korea, in 1970. He received the B.S. and M.S. degrees in electrical engineering from the Seoul National University, Seoul, Korea, in 1996 and 1998, respectively, and is currently working toward the Ph.D. degree at the Seoul National University.

His interests include the design of microwave circuits and linearization techniques for high-power amplifiers. 